Fixed Point Theory, 22(2021), No. 2, 603-624

DOI: $10.24193 /$ fpt-ro.2021.2.40

http://www.math.ubbcluj.ro/ nodeacj/sfptcj.html

\title{
FIXED POINTS AND TOPOLOGICAL PROPERTIES OF EXTENDED QUASI-METRIC SPACES
}

\author{
DEBASHIS DEY*, RAÚL FIERRO** AND MANTU SAHA*** \\ *Koshigram Union Institution, Burdwan, West Bengal, India \\ E-mail: debashismathdey@gmail.com \\ **Instituto de Matemáticas, Pontificia Universidad Católica de Valparaíso, \\ Casilla 4059, Valparaíso, Chile \\ E-mail: raul.fierro@pucv.cl \\ *** Department of Mathematics, The University of Burdwan, West Bengal, India \\ E-mail: mantusaha.bu@gmail.com
}

\begin{abstract}
In this paper, we introduce an extension of metric spaces, which includes $S$ spaces. Some properties of this topological structure are analyzed. Also, a non-compactness measure and condensing correspondences are defined for these type of spaces and natural results are obtained. Moreover, fixed point theorems for functions and correspondences satisfying certain Banach orbital condition are introduced and proved. These results are applied to contractions, which are defined by means of the extended quasi-metric, both for functions and correspondences.

Key Words and Phrases: Quasi-metric spaces, non-compactness measure, fixed point, contractions, correspondences, lower and upper semicontinuity, $b$-metric spaces.
\end{abstract}

2020 Mathematics Subject Classification: 47H08, 47H10, 54H15, 54G99.

Acknowledgment. This work was partially supported by Chilean Council for Scientific and Technological Research, under grant FONDECYT 1160868.

\section{REFERENCES}

[1] H. Aimar, B. Iaffei, L. Nitti, On the Macías-Segovia metrization of quasi-metric spaces, Rev. Un. Mat. Argentina, 41(1998), no. 2, 67-75.

[2] P. Alexandroff, P. Urysohn, Mémoire sur les espaces topologiques compactes, Verh. Akad. Wetensch, 14(1929), 1-91.

[3] V.T. An, L.Q. Tuyen, N.V. Dung, Stone-type theorem on b-metric spaces and applications, Topology Appl., 185-186(2015), 50-64.

[4] H. Aydi, A. Felhi, E. Karapinar, F.A. Alojail, Fixed points on quasi-metric spaces via simulation functions and consequences, J. Math. Anal., 9(2018), no. 2, 10-24.

[5] I.A. Bakhtin, The contraction mapping principle in almost metric spaces, Funct. Anal., Gos. Ped. Inst., Unianowsk, 30(1989), 26-37.

[6] S. Banach, Sur les opérations dans les ensembles abstraits et leur applications aux équations intégrales, Fund. Math., 3(1922), no. 1, 133-181.

[7] V. Berinde, Approximating fixed point of weak contractions using the Picard iteration, Nonlinear Analysis Forum, 9(2004), no. 1, 43-53. 
[8] N. Bourbaki, Elements of Mathematics, General Topology. Part 1, Hermann, Paris, 1966.

[9] S.K. Chatterjea, Fixed-point theorems, C.R. Acad. Bulgare Sci., 25(1972), 727-730.

[10] L.B. Ćirić, A generalization of Banach's contraction principle, Proc. Amer. Math. Soc., 45(1974), no. 2, 267-273.

[11] S. Czerwik, Contraction mapping in b-metric spaces, Acta Math. Inform. Universitatis Ostraviensis, 1(1993), 5-11.

[12] K. Darko, E. Karapinar, V. Rakočević, On quasi-contraction mappings of Ćirić and Fisher type via $\omega$-distance, Quaest. Math., 1(1993), 5-11.

[13] E. Estrada, The Structure of Complex Networks, Oxford University Press, 2011.

[14] R. Fierro, Noncompactness measure and fixed points for multi-valued functions on uniform spaces, Mediterr. J. Math., 15(2018), 95.

[15] R. Fierro, C. Martínez, E. Orellana, Weak conditions for existence of random fixed points, Fixed Point Theory, 12(2011), no. 1, 83-90.

[16] M. Furi, A. Vignoli, Fixed points for densifying mappings, Rend. Accad. Mat. Lincei, 47(1969), no. 6, 465-467.

[17] M. Gabeleh, C. Vetro, A new extension of Darbo's fixed point theorem using relatively MeirKeller condensing operators, Bull. Aust. Math. Soc., 98(2018), no. 2, 286-297.

[18] C. Horvath, Measure of non-compactness and multivalued mappings in complete metric topological vector spaces, J. Math. Anal. Appl., 108(1985), 403-408.

[19] R. Kannan, Some results on fixed points I, Amer. Math. Monthly, 76(1969), no. 4, 405-408.

[20] E. Karapinar, A. Fulga, M. Rashid, L. Shahid, H. Aydi, Large contractions on quasi-metric spaces with an application to nonlinear fractional differential equations, Math., $7(2019)$, no. 5, 11 pages.

[21] C. Kuratowski, Sur les espaces complets, Fund. Math., 15(1930), 301-309.

[22] R.A. Macias, C. Segovia, Lipschitz functions on spaces of homogeneous type, Adv. Math., 33(1979), 257-270.

[23] Q. Mahmood, A. Shoaib, M. Rasham, T. Arshadm, Fixed point results for the family of multivalued F-contractive mappings on closed ball in complete dislocated b-metric spaces, Math., $\mathbf{7}(2019), 7$ pages.

[24] M. Mehran, M. Egerstedt, Graph Theoretic Methods in Multiagent Networks, Princeton University Press, 2010.

[25] D. Mitrea, I. Mitrea, M. Mitrea, S. Monniaux, Groupoid Metrization Theory, with Applications to Quasi-Metric Spaces and Functional Analysis, Springer, New York, 2013.

[26] S.B. Nadler, Multivalued contraction mappings, Pacific J. Math., 30(1969), no. 2, 475-487.

[27] H.K. Nashine, R. Arab, R.P. Agarwal, M. Sen, Positive solutions of fractional integral equations by the technique of measure of noncompactness, J. Inequal. Appl., 2017(2017), no. 225, 17 pages.

[28] M. Paluszynski, K. Stempak, On quasi-metric and metric spaces, Proc. Amer. Math. Soc., 137(2009), no. 12, 429-439.

[29] S. Reich, Some remarks concerning contraction mappings, Canad. Math. Bull., 4(1971), 121124.

[30] S. Sedghi, N. Shobe, A. Aliouche, A generalization of fixed point theorems in S-metric spaces, Mat. Vesnik, 64(2012), no. 3, 258-266.

[31] D. Sekman, N.E. Bouzara, V. Karakaya, n-tuplet fixed points of multivalued mappings via measure of noncompactness, Commun. Optim. Theory, 2017(2017), 13 pages, 2017.

[32] J. Vujaković, H. Aydi, S. Radenović, A. Mukheimer, Some remarks and new results in ordered partial b-metric spaces, Math., $\mathbf{7}(2019)$, no. 4, 7 pages.

Received: October 23, 2019; Accepted: February 7, 2020. 
